\title{
Color-Based Classification of Natural Rock Images Using Classifier Combinations
}

\author{
Leena Lepistö, Iivari Kunttu, and Ari Visa \\ Tampere University of Technology, Institute of Signal Processing, \\ P.O. Box 553, FI-33101 Tampere, Finland \\ \{Leena.Lepisto, Iivari.Kunttu, Ari.Visa\}atut.fi \\ http://www.tut.fi/
}

\begin{abstract}
Color is an essential feature that describes the image content and therefore colors occurring in the images should be effectively characterized in image classification. The selection of the number of the quantization levels is an important matter in the color description. On the other hand, when color representations using different quantization levels are combined, more accurate multilevel color description can be achieved. In this paper, we present a novel approach to multilevel color description of natural rock images. The description is obtained by combining separate base classifiers that use image histograms at different quantization levels as their inputs. The base classifiers are combined using classification probability vector (CPV) method that has proved to be an accurate way of combining classifiers in image classification.
\end{abstract}

\section{Introduction}

Image classification is an essential task in the field of image analysis. The classification is usually based on a set of visual features extracted from the images. These features may characterize for example colors or textures occurring in the images. Most of the real-world images are seldom homogenous. Especially, different kinds of natural images have often non-homogenous content. The division of natural images like rock, stone, clouds, ice, or vegetation into classes based on their visual similarity is a common task in many machine vision and image analysis solutions. In addition to nonhomogeneities, the feature patterns can be also noisy and overlapping. Due to these reasons, different classifiers may classify the same image in different ways. Hence, there are differences in the decision surfaces, which lead to variations in classification accuracy. However, it has been found that a consensus decision of several classifiers can give better accuracy than any single classifier [1],[8],[9]. This fact can be easily utilized in the classification of real-world images.

The goal of combining classifiers is to form a consensus decision based on opinions provided by different base classifiers. Duin [5] presented six ways, in which consistent set of base classifiers can be generated. In the base classifiers, there can be differences in initializations, parameter choices, architectures, classification principle, training sets, or feature sets. Combined classifiers have been applied to several classification tasks, for example to face recognition [15], person identification [3] and fingerprint verification [7]. Theoretical framework for combining classifiers is provided in [8]. 
In the image classification, several types of classifier combination approaches can be used. In our previous work [11],[13] we have found that different feature types can be easily and effectively combined using classifier combinations. In practice, this is carried out by making the base classification for each feature type separately. The final classification can be obtained based on the combination of separate base classification results. This has proved to be particularly beneficial in the case of nonhomogenous natural images [11],[13]. Hence, the non-homogenous properties of individual features do not necessarily affect directly on the final classification. In this way, each feature has its own affect on the classification result.

Rock represents typical example of non-homogenous natural image type. This is because there are often strong differences in directionality, granularity, or color of the rock texture, even if the images represented the same rock type [11]. Moreover, rock texture is often strongly scale-dependent. Different spatial multiscale representations of rock have been used as classification features using Gabor filtering [12]. However, the scale dependence of the rock images can be used also in another way, using color quantization. It has been found that different color features can be found from the rock images using different numbers of quantization levels. Hence, by combining the color representation at several levels, a multilevel color representation can be achieved. For this kind of combination, a classifier combination method can be used. In this paper, we present our method to make a classifier combination that is used to produce this multilevel color representation. The rest of this paper is organized as follows. Section two presents the main principle of classifier combinations as well as our method for that purpose. In section three, the principle of multilevel color representation is presented. The classification experiments with natural rock images are presented in section four. The obtained results are discussed in section five.

\section{Classifier Combinations in Image Classification}

The idea of combining classifiers is that instead of using single decision making theme, classification can be made by combining opinions of separate classifiers to derive a consensus decision [8]. This can increase classification efficiency and accuracy. In this section, methods for combining separate classifiers are presented. Furthermore, we present our approach to make a probability-based classifier combination.

\subsection{Methods for Combining Classifiers}

The general methods for combining classifiers can be roughly divided into two categories, voting-based methods and the methods based on probabilities. The voting-based techniques are popularly used in pattern recognition [10],[14]. In the voting-based classifier combinations, the base classifier outputs vote for the final class of an unknown sample. These methods do not require any additional information, like probabilities from the base classifiers. Voting has proved to be a simple and effective method for combining classifiers in several classification problems. Also in the comparisons with the methods presented by Kittler et al., voting-based methods have given relatively accurate classification results [8]. Lepistö et al. [11] presented a method for combining 
classifiers using classification result vectors (CRV). In this approach, the class labels provided by the base classifiers are used as a feature vector in the final classification, and hence the result is not based on direct voting. CRV method outperformed voting method in the classification experiments. In [13] an unsupervised variation of CRV was presented and compared to other classifier combinations.

Recently, the probability-based classifier combination strategies have been popularly used in pattern recognition. In these techniques, the final classification is based on the a posteriori probabilities of the base classifiers. Kittler et al. [8] presented several common strategies for combining base classifiers. These strategies are e.g. product rule, sum rule, max rule, min rule, and median rule. All these rules are based on the statistics computed based on the probability distributions provided by the base classifiers. In [8], the best experimental results have been obtained using sum and median rules. Theoretical comparison of the rules has been carried out in [9]. Also Alkoot and Kittler [1] have compared the classifier combination strategies.

\subsection{Classification Probability Vector Method}

Our previous method for combining classifiers combined the outputs of the base classifiers into a feature vector that is called classification result vector (CRV) [11]. However, CRV method uses only the class labels provided by the base classifiers, and ignores their probabilities. In this paper, we use the probability distributions of the separate base classifiers as features in the final classification.

In general, in the classification problem a pattern $S$ is to be assigned to one of the $m$ classes $\left(\omega_{1}, \ldots, \omega_{m}\right)$ [8]. We assume that we have $C$ classifiers each representing a particular feature type and we denote the feature vector used by each classifier by $f_{i}$. Then each class $\omega_{k}$ is modeled by the probability density function $p\left(f_{I} \mid \omega_{k}\right)$. The priori probability of occurrence of each class is denoted $P\left(\omega_{k}\right)$. The well-known Baeysian decision theory [4],[8] defines that $S$ is assigned to class $\omega_{j}$ if the a posteriori probability of that class is maximum. Hence, $S$ is assigned to class $\omega_{k}$ if:

$$
P\left(\omega_{j} \mid f_{1}, \ldots, f_{C}\right)=\max _{k} P\left(\omega_{k} \mid f_{1}, \ldots, f_{C}\right)
$$

However, the probabilities of all the other classes than $\omega_{j}$ have also significance in classification. They are particularly interesting when the pattern $S$ is located near the decision surface. Therefore, we focus on the whole probability distribution $p\left(f_{i} \mid \omega_{1}, \ldots, \omega_{m}\right)$ provided by each classifier. Hence, if the probability is defined for each $m$ class, the obtained probability distribution is a $C$ by $m$ matrix for each pattern $S$. This matrix is used as a feature vector in the final classification, and it is called

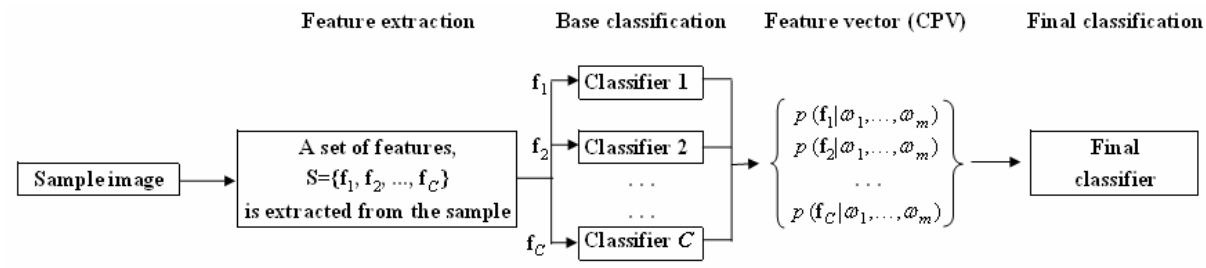

Fig. 1. The outline of the CPV classifier combination method 
classification probability vector (CPV). In the final classification, the images with similar CPV's are assigned into same classes. The outline of the CPV method is presented in figure 1 .

The common probability-based classifier combinations [8] are used to calculate some statistics based on the probability distributions provided by the base classifiers. In contrary to them, CPV method uses the whole probability distribution as a feature vector in the final classification. The CPV method utilizes the fact that the separate base classifiers classify similar samples in the similar way, which leads to a similar probability profile. The final classification is based merely on the similarity between the probabilities of the base classifiers. Hence, in contrary to voting, in the CPV method the base classifier outputs (class labels) do not directly affect the final classification result.

When image classification is considered, the CPV method has several advantages. CPV method considers each visual feature of the images in the base classifiers separately. In the final classification, the probability distributions are employed instead of features. This way, the individual features do not directly affect the final classification result. Therefore, classification result is not sensitive to variations and nonhomogeneities of single images.

\section{Multilevel Color Representation Using Quantization}

\subsection{Color Image Representation}

In digital image representation, an image has to be digitized in two manners, spatially (sampling) and in amplitude (quantization) [6]. The use of spatial resolutions is common in different texture analysis and classification approaches, whereas the effect of quantization is related to the use of image color information. The quantization can be applied to different channels of a color image. Instead of using common RGB color space, the use of HSI space has found to be effective, because it corresponds to the human visual system [16].

A common way of expressing the color content of an image is the use of image histogram. Histogram is a first-order statistical measure that expresses the color distribution of the image. The length of the histogram vector is equal to the number of the quantization levels. Hence the histogram is a practical tool for describing the color content at each level. Histogram is also a popular descriptor in color-based image classification, in which images are divided into categories based on their color content.

\subsection{Multilevel Classification}

The classifier combination tools presented in section two provide a straightforward tool for making a histogram-based image classification at multiple levels. Hence the histograms at selected quantization levels and color channels are used as separate input features. Each feature is then classified separately at base classification. After that, the base classification results are combined to form the final classification. This way the final classifier uses multilevel color representation as classifying feature. 

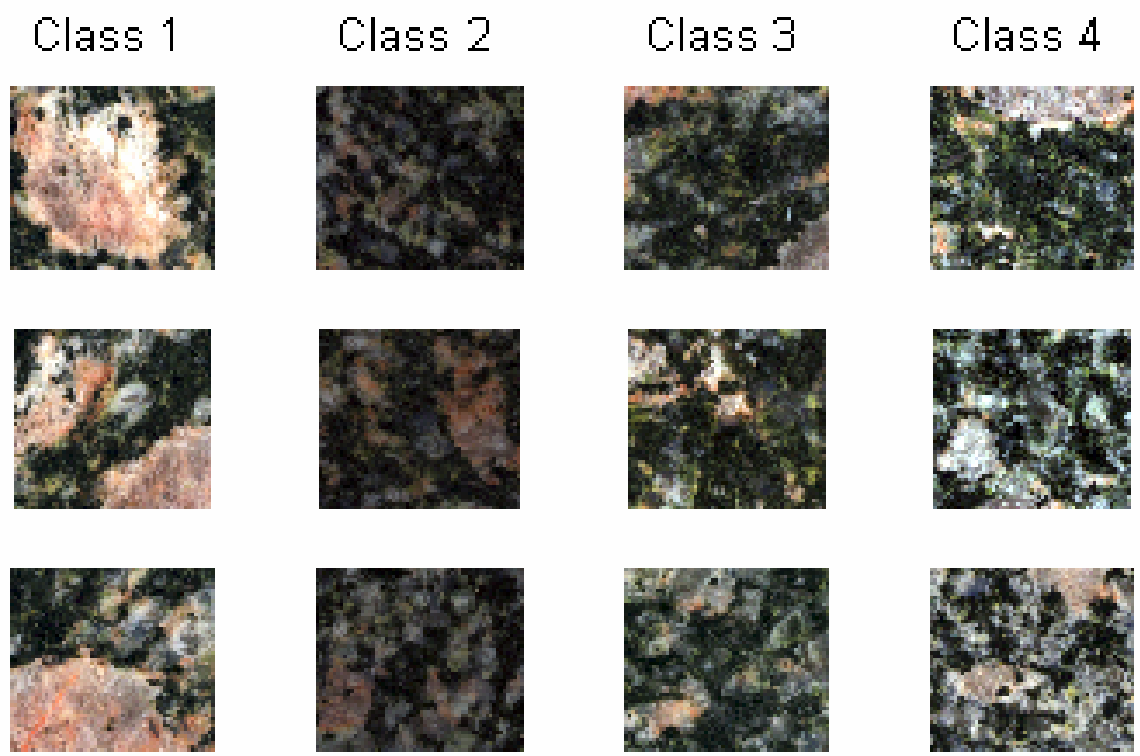

Fig. 2. Three example images from each rock type in the testing database

\section{Experiments}

In this section, we present the classification experiments using rock images. The purpose of the experiments is to show that an accurate multilevel color representation is achievable using classifier combinations. We also compare the CPV method to other classifier combination approaches.

\subsection{Rock Images}

The experiments in this paper are focused on non-homogenous natural image data that is represented by a database of rock images. There is a practical need for methods for classification of rock images, because nowadays rock and stone industry uses digital imaging for rock analysis. Using image analysis tools, visually similar rock texture images can be classified. Another application field for rock imaging is geological research work, in which the rock properties are inspected using borehole imaging. Different rock layers can be recognized and classified from the rock images based on e.g. the color and texture properties of rock. The degree of non-homogeneity in rock is typically overwhelming and therefore, there is a need for an automatic classifier that is capable of classifying the borehole images into visually similar classes. The testing database consists of 336 images that are obtained by dividing large borehole images into parts. These images are manually divided into four classes by an expert. In classes 1-4, there are 46, 76, 100, and 114 images in each class, respectively. Figure 2 presents three example images of each four class. 


\subsection{Classification Experiments}

In the experimental part, the principle for classification was selected to be $k$-nearest neighbor $(k-\mathrm{NN})$ method in base classification and final classification. Barandela et al. [2] have proved that nearest neighbor principle is efficient and accurate method to be used in classifier combinations. Classification results are obtained using leave one out validation principle [4]. The distance metrics for the comparison of histograms in the base classification was selected to be $L_{1}$ norm. In the CPV method, the final classifier used $L_{2}$ norm (Euclidean distance) to compare CPV:s.

The histograms were calculated for the database images in HSI color space. In the classification experiments we used hue $(\mathrm{H})$ and intensity (I) channels, which have been proved to be effective in color description of rock images [12]. The hue and intensity histograms were calculated for the images quantized to 4, 16, and 256 levels. Hence the number of the features was six. The classification was carried out using values of $k$ varying between 1 and 15. In the first experiment, the classification rate of CPV method was compared to that of separate base classifiers which use different histograms. In this comparison, also the classification accuracy all the histograms combined into a single feature vector was tested. The average classification rates are presented in figure 3 as a function of $k$. The second experiment measured the classification accuracy of different classifier combination strategies compared to CPV method. The idea of this experiment was to combine the six base classifiers that use different histograms as input features. In this case, CPV was compared to the most usual probability-based classifier combinations, sum, max and median rules [8]. Product rule is not included into comparison, because the probability estimates of

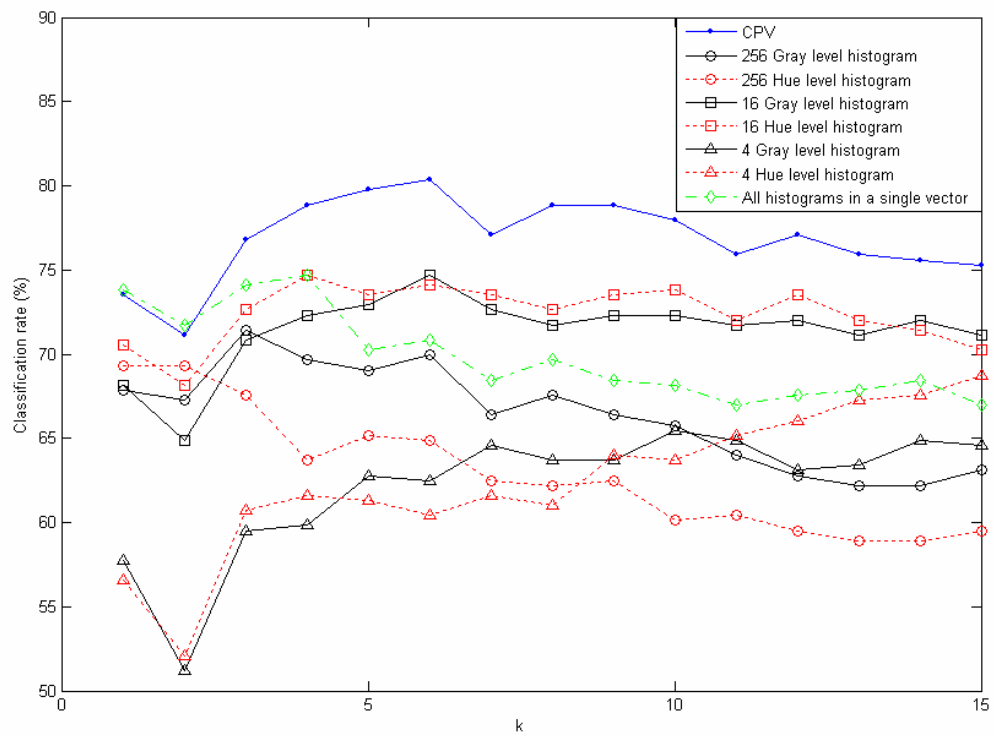

Fig. 3. The average classification rates of the rock images using base classifiers that use different histograms and the classifier combination (CPV) 
$k$-NN classifiers are sometimes zero, which may corrupt the result. In addition to the selected probability-based combination methods, we used also majority voting and our previously introduced CRV method [11] in the comparison. Figure 4 presents the results of this comparison with $k$ varying between 1 and 15 .

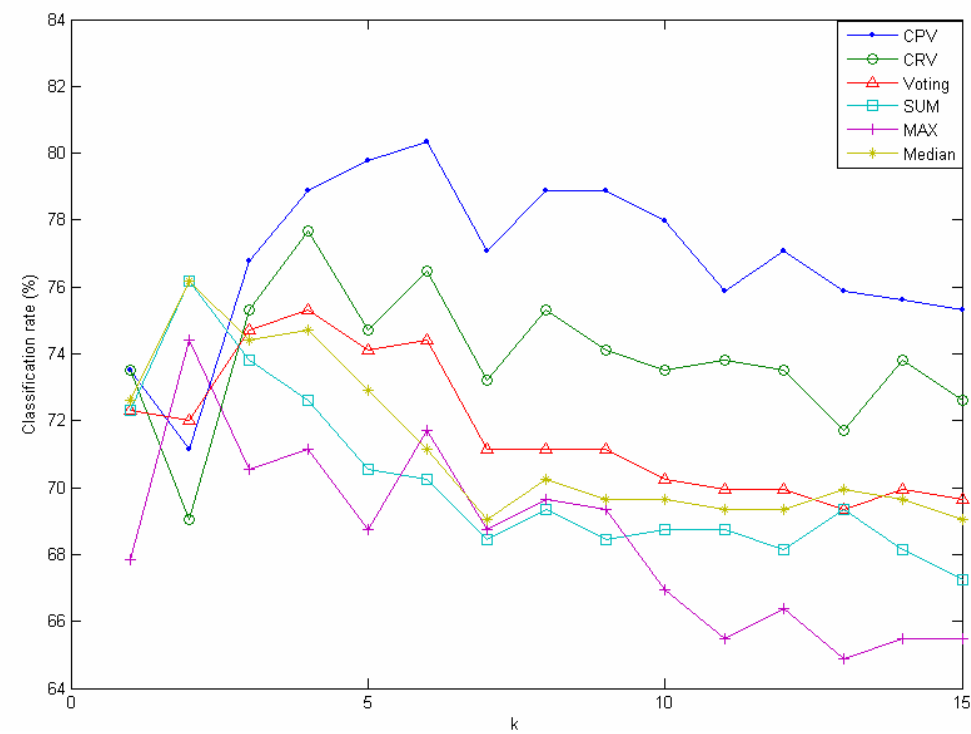

Fig. 4. The average classification rates of the rock images using different classifier combinations

\subsection{Results}

The results presented in figure 3 show that using CPV the classification accuracy is clearly higher than that of any single base classifier. The performance of CPV is also compared to the alternative approach, in which all the histograms are collected into a single feature vector. This vector is very high dimensional (552 dimensions), and its performance is significantly lower than in the case of CPV. This observation gives the reason for the use of classifier combinations in the image classification. That is, different features can be combined by combining their separate base classifiers rather than combining all the features into a single high dimensional feature vector in classification. This way, also the "curse of dimensionality" can be avoided.

The results of the second experiment presented in figure 4 show that CPV method outperforms the other classifier combinations in the comparison with a set of rock images. Also the CRV [11] method gives relatively good classification performance. Only with small values of $k \mathrm{CPV}$ is not accurate one. This is due to the probability distributions used by CPV are able to effectively distinguish between image classes only when more than three nearest neighbors are considered in $k$-NN algorithm. 


\section{Discussion}

In this paper, we presented a method for combining classifiers in the classification of real rock images. Due to their non-homogenous nature, the classification of them is a difficult task. We presented a method for an effective multilevel color representation using our classifier combination strategy, classification probability vector (CPV). In CPV method, the feature vector that describes the image content is formed using the probability distributions of separate base classifiers. The probabilities provided by the base classifiers form a new feature space, in which the final classification is made. Hence the final classification depends on the metadata of the base classification, not the image features directly. This way the non-homogeneities of individual features do not have direct impact on the final result.

In the color-based image classification, like in image classification in general, it is often beneficial to combine different visual features to obtain the best possible classification result. Therefore, classifiers that use separate feature sets can be combined. In this study this feature combination approach was applied to color histograms with different numbers of bins. By combining the histograms using classifier combinations, a multilevel color representation was achieved. The experimental results showed that this representation outperforms any single histogram in classification. Furthermore, CPV method also gives better classification accuracy than any other classifier combination in the comparison.

\section{Acknowledgement}

The authors wish to thank Saanio \& Riekkola Oy for the rock image database used in the experiments.

\section{References}

1. Alkoot, F.M., Kittler, J.: Experimental evaluation of expert fusion strategies, Pattern Recognition Letters, Vol. 20 (1999) 1361-1369

2. Barandela, R., Sánchez, J.S., Valdovinos, R.M.: New applications of ensembles of classifiers, Pattern Analysis \& Applications, Vol. 6 (2003) 245-256

3. Brunelli, R., Falavigna, D.: Person Identification Using Multiple Cues, IEEE Transactions on Pattern Analysis and Machine Intelligence, Vol. 17 (1995) 955-966

4. Duda, R.O., Hart, P.E., Stork, D.G.: Pattern Classification, $2^{\text {nd }}$ ed., John Wiley \& Sons, New York (2001)

5. Duin, R.P.W.: The Combining Classifier: to Train or Not to Train, In: Proceedings of $16^{\text {th }}$ International Conference on Pattern Recognition, Vol. 2 (2002) 765-770

6. Gonzales, R.C., Woods, R.E.: Digital Image Processing, Addison Wesley, 1993.

7. Jain, A.K., Prabhakar, S., Chen, S.: Combining Multiple Matchers for a High Security Fingerprint Verification System, Pattern Recognition Letters, Vol. 20 (1999) 1371-1379

8. Kittler, J., Hatef, M., Duin, R.P.W., Matas J.: On Combining Classifiers, IEEE Transactions on Pattern Analysis and Machine Intelligence, Vol. 20 (1998) 226-239

9. Kuncheva, L.I.: A Theoretical Study on Six Classifier Fusion Strategies, IEEE Transactions on Pattern Analysis and Machine Intelligence, Vol. 24 (2002) 281-286 
10. Lam, L., Suen, C.Y.: Application of majority voting to pattern recognition: An analysis of the behavior and performance, IEEE Transactions on Systems, Man, and Cybernetics, Vol. 27 (1997) 553-567

11. Lepistö, L., Kunttu, I., Autio, J., Visa, A.: Classification of Non-homogenous Textures by Combining Classifiers, Proceedings of IEEE International Conference on Image Processing, Vol. 1 (2003) 981-984

12. Lepistö, L., Kunttu, I., Autio, J., Visa, A.: Classification Method for Colored Natural Textures Using Gabor Filtering, In: Proceedings of $12^{\text {th }}$ International Conference on Image Analysis and Processing (2003) 397-401

13. Lepistö, L., Kunttu, I., Autio, J., Visa, A.: Combining Classifiers in Rock Image Classification - Supervised and Unsupervised Approach, In: Proceedings of Advanced Concepts for Intelligent Vision Systems, (2004) 17-22

14. Lin, X., Yacoub, S., Burns, J., Simske, S.: Performance analysis of pattern classifier combination by plurality voting, Pattern Recognition Letters, Vol. 24 (2003) 1959-1969

15. Lu, X., Wang, Y., Jain, A.K.: Combining Classifiers for Face Recognition, In: Proceedings of International Conference on Multimedia and Expo, Vol. 3 (2003) 13-16

16. Wyszecki, G., Stiles, W.S.: Color Science, Concepts and Methods, Quantitative Data and Formulae, $2^{\text {nd }}$ Edition, John Wiley \& Sons (1982) 